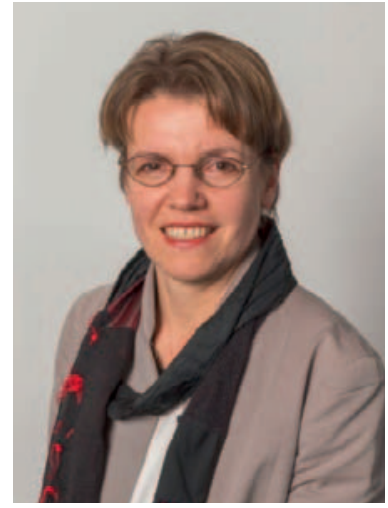

Dr. sc. nat. Beatrix Falch, Vizepräsidentin SMGP, Zürich, Schweiz

\section{Spannungsfeld: Arzneipflanzen - Nahrungspflanzen}

Je mehr Pflanzen unser täglicher Speiseplan enthält, als umso gesünder gilt unsere Ernährung. Diesem Grundsatz folgend ist auch die Lebensmittelpyramide aufgebaut.

Was wir essen, soll aber nicht nur gesund sein, sondern uns auch gesund erhalten. Die vielen Diäten und wechselnden Ernährungstrends geben Zeugnis davon, welche Bedeutung der Ernährung bezüglich Krankheitsprävention beigemessen wird. So nimmt in jüngster Zeit die Zahl an Personen zu, die sich vegan ernähren, also nur Pflanzen und aus Pflanzen hergestellte Nahrungsmittel zu sich nehmen. Dieser Bewegung ist wahrscheinlich auch die derzeitige Beliebtheit von Grünsäften - oder auch «Green Smoothies» genannt - zu verdanken.

Dass Nahrungspflanzen mehr als nur einen nährenden Wert haben, ist ein jahrtausendealtes Wissen, denn schon Hippokrates sagte: «Eure Nahrungsmittel sollen Eure Heilmittel und Eure Heilmittel Eure Nahrungsmittel sein!»

Vor einigen Jahren war das Schlagwort «Functional Food» in aller Munde. Die gesunderhaltende und präventive Wirkung von Pflanzen und ihren sekundären Inhaltsstoffen wollte man sich zunutze machen, indem man traditionelle Nahrungsmittel mit pflanzlichen Extrakten anreichern wollte. Die dank zahlreicher Untersuchungen bekannten gesundheitsfördernden Wirkungen von sekundären pflanzlichen Inhaltsstoffen, wie Flavonoiden und anderen Polyphenolen, Senfölglykosiden, ungesättigten Fettsäuren, Sterolen, präbiotisch wirksamen Kohlenhydraten und vielen mehr, sollten die gängigen Nahrungsmittel noch wertvoller und vor allem gesünder machen.
So verlockend es klingen mag, aber konzentrationsförderndes Brot, da angereichert mit Vitaminen und Omega3-Fettsäuren, glücklich machende Joghurts dank eines $\mathrm{Zu}$ satzes von Johanniskrautextrakten oder krebshemmende Wurstwaren aufgrund des Zusatzes von Brokkoliextrakten haben als «funktionelle Nahrungsmittel» letztendlich den Durchbruch nicht geschafft. Der Mensch scheint mehrheitlich in seinen Essensgewohnheiten eher traditionell und konservativ orientiert und relativ wenig experimentierfreudig zu sein.

Dagegen haben sich sogenannte Nahrungsergänzungsmittel auf dem Markt etabliert. Diese Präparate, die keine Medikamente, aber auch keine Nahrungsmittel sind, werden zusätzlich zur täglichen Nahrung eingenommen, um die Körperfunktionen zu erhalten, zu stärken oder zu verbessern und um Krankheiten vorzubeugen.

Dieser Markt wächst. Einerseits, weil wir alle möglichst lange gesund bleiben und jung aussehen möchten; andererseits, weil die Zulassungsbedingungen für pflanzliche Arzneimittel sehr aufwendig und vor allem teuer sind. Rein regulatorisch gesehen ist es daher viel einfacher und kostengünstiger, ein pflanzliches Nahrungsergänzungsmittel auf den Markt zu bringen als ein pflanzliches Arzneimittel. Doch so einfach ist die regulatorische Abgrenzung von Nahrungsergänzungsmitteln und Heilmitteln in der Praxis nicht. Und auch für die Konsumenten ist es schwierig zu unterscheiden, ob sie nun ein Arzneimittel oder ein Nahrungsergänzungsmittel zu sich nehmen, da die Grenzen zwischen gesundheitsfördernden Anpreisungen und Heilanpreisungen für den Laien nicht immer klar erkennbar sind.

Und so stellen sich einige grundsätzliche Fragen:

- Was macht letztendlich eine Nahrungspflanze zu einer Heil- oder Arzneipflanze?

- Ist es in Anlehnung an Paracelsus die Dosis und/oder die Zubereitungsweise?

- Ist es überhaupt wünschenswert, wenn pflanzliche Extrakte zunehmend als Nahrungsergänzungsmittel auf den Markt kommen?

- Kann man sich mit einer Diät, die aus vielen Pflanzen mit nachgewiesenen medizinischen Wirkungen besteht, vor Krankheiten schützen oder sogar Krankheiten heilen?

- Wenn ja, wie müsste eine solche pflanzenbetonte Diät konkret aussehen?

- Wenn diese Art von Krankheitsprävention und Heilung gelingen würde, braucht es dann überhaupt noch Arzneimittel?

\section{KARGER}

() 2016 S. Karger GmbH, Freiburg

Fax +497614520714 
- Oder sind die Wirkungen der Nahrungspflanzen doch eher unspezifisch und daher «nur» gesunderhaltend?

- Kann mit Pflanzen erst durch die Herstellung spezieller Extrakte eine spezifische Wirkrichtung erzielt werden?

- Müsste nicht eine solche Forschung staatlich gefördert werden, welche die Verwendungsweise von Pflanzen zur Gesunderhaltung, Krankheitsprävention und Therapie genauer untersucht?

- Würden wir nicht enorme Gesundheitskosten einsparen, wenn wir gezielt pflanzliche Extrakte täglich einnehmen würden?

Diese Fragen sollen zur Diskussion und kritischen Auseinandersetzung anregen, in welche Richtung unser Gesundheitswesen und die Forschung im Gesundheitsbereich in den nächsten Jahren und Jahrzehnten gehen sollen.

Das Potenzial der Pflanzen, sei es als Nahrungs- oder als Arzneipflanzen, wurde bisher völlig unterschätzt. Daher ist es absolut notwendig, dass sich Hochschulen, Politik wie auch Behörden bewusst werden, welche Dringlichkeit nicht nur in Anbetracht der Kostenexplosion im Gesundheitswesen besteht, neue therapeutische Wege zu erforschen.

Schon heute kann in der ärztlichen Praxis und in der pharmazeutischen Beratung das Potenzial der Arznei- und Nahrungspflanzen genutzt werden - wer sich neu mit dieser Materie befasst, staunt immer wieder, wie viele wissenschaftlich belegte Kenntnisse vorhanden sind und von welchen Erfolgen erfahrene Kolleginnen und Kollegen bei der Arbeit mit Pflanzen im Sinne der Phytotherapie, aber auch im Sinne der Ernährungsberatung berichten.

Die Schweizerische Medizinische Gesellschaft für Phytotherapie (SMGP) greift an ihrer diesjährigen Tagung am 10. November in Baden die oben ausgeführte Thematik auf. Denn: Die Ernährung ist ein gesamtmedizinisches Problem, das breitere Kreise interessiert. Somit ist die Tagung die ideale Gelegenheit, sich zusätzlich zur Phytotherapie mit der Ernährung auseinanderzusetzen. Seien Sie, liebe Leserinnen und Leser versichert, dass ein spannendes wissenschaftliches Programm in Baden auf Sie wartet. 